OPEN ACCESS

\section{Ways to make polarized antiproton beams}

To cite this article: D Grzonka et al 2011 J. Phys.: Conf. Ser. 295012171

View the article online for updates and enhancements.

\section{Related content}

- Antiproton beam polarizer using a dense
polarized target
Bogdan Wojtsekhowski
- Spin-filtering at COSY
Christian Weidemann and the PAX
collaboration
- Spin dependence of the antinucleon-
$\frac{\text { nucleon interaction }}{\text { Johann Haidenbauer }}$




\title{
Ways to make polarized antiproton beams
}

\author{
D Grzonka ${ }^{1}, K_{\text {Kilian }}{ }^{1}$, D Möhl ${ }^{2}$, W Oelert ${ }^{1}$ and T Sefzick ${ }^{1}$ \\ ${ }^{1}$ IKP, Forschungszentrum Jülich, 52425 Jülich, Germany \\ ${ }^{2}$ CERN, CH-1211 Geneva 23, Switzerland \\ E-mail: d.grzonka@fz-juelich.de
}

\begin{abstract}
For producing polarized antiproton beams the so called spin filter method is normally discussed. The method has been proven by the FILTEX collaboration with a proton beam and detailed studies are presently performed by the PAX collaboration at COSY. A well known source for polarized antiprotons is the antilambda decay. It was used in the only experiment with polarized antiprotons so far at FERMILAB.

Furthermore the antiproton production process itself, if showing polarization, would be by far the simplest and best way to get polarized $\bar{p}$ beams because one could use the existing antiproton facility. Up to now $\bar{p}$ production has never been investigated in detail.
\end{abstract}

\section{Introduction}

A dedicated facility for low energy antiproton research (LEAR) was built at CERN in the 1980s. Many proposals for experiments with antiprotons came up but not all suggestions could be realized at LEAR and some of these propositions were updated in view of the upcoming antiproton facility at FAIR including the preparation of polarized antiproton beams.

The spin filter method in a storage ring was first suggested in 1968 in order to polarize high energy protons in the CERN ISR [1]. It is based on the depletion of one spin component due to the spin dependent interaction if a stored beam passes a polarized target. For filtering antiprotons at lower momenta it was pointed out later that the new technique of phase space cooling is mandatory and the relevant parameters were presented [2],[3]. The feasibilty of the method depends on the magnitude and the difference of the spin-spin singlet and triplet cross sections. In 1993 a feasibility study of the filter method with phase space cooling has been performed with a proton beam on polarized protons at the TSR in Heidelberg showing clearly the buildup of polarization [4]. For antiprotons the filter method with cooling should also work if one can find any filter interaction with both large spin-spin dependence and cross section. For several years the favorite idea was the spin transfer from polarized electrons to antiprotons. This spin transfer does not work as shown by the PAX collaboration in an experiment at COSY with polarized protons [5]. High polarization effects exist in interactions of polarized photons with antiprotons [6]. Problems here are the small cross sections and the low intensity and quality of photon beams. Presently the PAX collaboration is investigating the antiproton interaction with polarized protons as filter [7],[5],[8]. Till now there are no data for the spin-spin dependence of the total $\bar{p} p$ cross section available. From theoretical predictions one expects that longitudinal polarization effects are larger than transversal effects [9], [8], [10],[11]. The consequence might be that a Siberian snake is needed in the filter synchrotron. 
The PAX collaboration estimates for the final polarization of an antiproton beam, assuming an ideal machine where the Coulomb losses are dominated by the target, values between 20 $40 \%$ at the maximum figure of merit $\left(p^{2} * I\right)$ after two beam life times $\left(\tau_{\text {beam }} \sim\right.$ days $)$ with an intensity reduction to $N=0.14 N_{0}[7],[8]$.

This estimation based on theoretical predictions which differ in a wide range depending on the model for the $\bar{p} p$ scattering process. Calculations with a Paris potential for $\bar{p}$ energies up to $100 \mathrm{MeV}$ result in achievable polarizations of $8 \%(12 \%)$ for $P_{\perp}$ at $60 \mathrm{MeV}\left(P_{\|}\right.$at 100 $\mathrm{MeV})[10]$ while the Nijmegen potential gives values up to $20 \%(40 \%)$ for $P_{\perp}\left(P_{\|}\right)$above $200 \mathrm{MeV}$ [11]. Experimental $\bar{p} p$ scattering data are needed. A proposal for such studies at the CERN / AD has been submitted by the PAX collaboration [8]. Besides the filter method other ideas how to polarize antiprotons came up using the Stern-Gerlach effect, dynamic nuclear polarization, channeling, spin flip processes, interaction with polarized photons, antihydrogen atoms, or hyperon decay. A first collection of the various ideas has been presented at a workshop in Bodega Bay in 1985 [12] and overviews can be found e.g., in [13],[14],[15].

\section{Antihyperon decay}

A source of antiprotons with large polarization is the decay of the $\bar{\Lambda}$ hyperons. The parity violating weak decay of $\bar{\Lambda}$ with a branching ratio of $0.639( \pm 0.005)$ into $\bar{p} \pi^{+}$results in a $\bar{p}$ helicity of $64.2( \pm 1.3) \%$ in the $\bar{\Lambda}$ rest frame [16]. By measuring the direction and momenta of the $\bar{\Lambda}$, the $\bar{p}$, and the $\pi^{+}$in the laboratory system, the decay kinematics can be reconstructed and the transversal and longitudinal antiproton polarization components in the lab. system for each event can be determined. This was performed at FERMILAB in the only experiment with polarized antiprotons so far [17]. In these studies a high energy proton beam of 800 $\mathrm{GeV} / \mathrm{c}$ momentum has been used to produce the antihyperons $(\bar{\Lambda})$ and their decay antiprotons with momenta around $200 \mathrm{GeV} / \mathrm{c}$. At the FAIR facility the beam energies will be much lower with antihyperon and antiproton momenta in the few $\mathrm{GeV} / \mathrm{c}$ region. With a detection system like the PS185 experiment at LEAR [18] the $\bar{\Lambda}$ charged decay products can be identified and measured precisely in direction and momentum. By introducing a secondary target these polarized antiprotons can be used for reaction studies. In figure 1 the decay kinematics of a $\bar{\Lambda}$ (resulting from a $\bar{p} p \rightarrow \bar{\Lambda} \Lambda$ reaction) at $3.5 \mathrm{GeV} / \mathrm{c} \bar{p}$ beam momentum is shown. By the Lorentz boost a rather large transverse polarization is projected out from the $\bar{p}$ helicity.

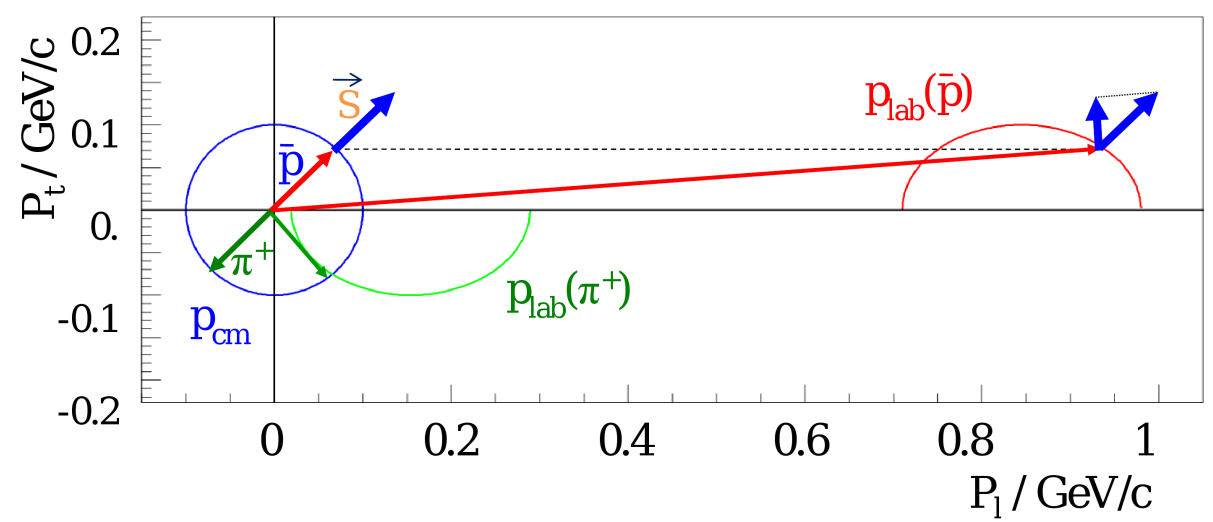

Figure 1. Kinematics of the $\bar{\Lambda}$ decay into $\bar{p} \pi^{+}$for a $\bar{\Lambda}$ momentum at $1 \mathrm{GeV} / \mathrm{c}$. The $\bar{p}$ helicity in the $\bar{\Lambda}$ decay system is projected into a transversal vector polarization component in the laboratory system. 


\section{Polarization in antiproton production ?}

Another possible source of polarized antiprotons may be the production process itself. It is typically done by bombarding a solid target with high momentum protons. At CERN the beam momentum is $26 \mathrm{GeV} / \mathrm{c}$ and the number of collected antiprotons is around one per $10^{6}$ beam protons using a $6 \mathrm{~cm}$ long iridium target. The $\bar{p}$ momentum spectrum peaks at around $3.5 \mathrm{GeV} / \mathrm{c}$ which is consistent with a pure phase space distribution in a quasi-free $p$ - nucleon interaction into a four particle final state: $p N \rightarrow p N \bar{p} p$. The basic process is a creation of a baryonantibaryon pair out of collisional energy. There could be production of polarized antiprotons, but no experimental studies have been performed so far. If transverse polarization occurs (figure 2 ) then a polarized beam can be prepared in a rather simple and cheap way by blocking up and down events, one side of the angular distribution, and the pure $\mathrm{S}$ wave region around 0 degree $(<50 \mathrm{mrad})$. A simple modification of the extraction beam line with absorbers is sufficient (figure 2). Of course one has to avoid depolarizing resonances in the cooler synchrotron, which for the present $\mathrm{AD}$ optics seems manageable.

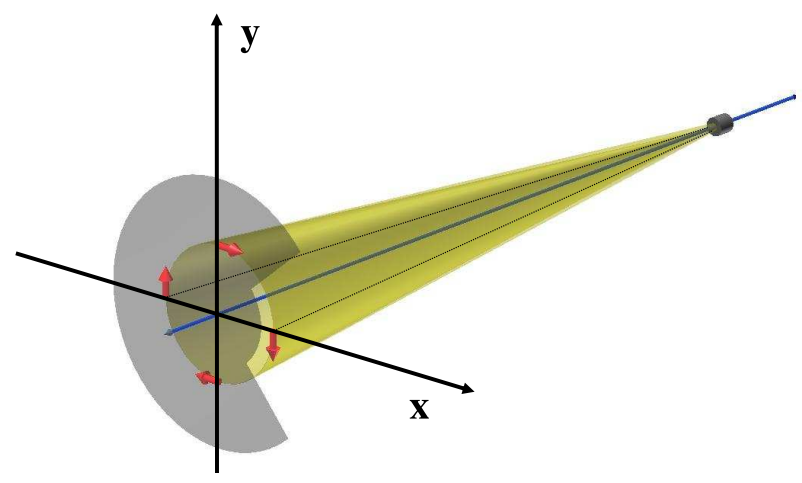

Figure 2. The red arrows indicate the polarization vector for a given production polar angle $\theta$. Sketched are the required cuts in the produced $\bar{p}$ distribution in order to select an antiproton beam with transverse polarization.

In order to measure the polarization of the produced antiprotons, a double scattering experiment is necessary where the antiprotons scatter on an analyzer target behind which a detector system is placed to determine the asymmetry. Known and calculable is the analyzing power in the $\bar{p} p$ elastic scattering in the Coulomb nuclear interference $(\mathrm{CNI})$ region. There is a maximum asymmetry of $4.5 \%$ at a momentum transfer of $t=-0.0037 \mathrm{GeV} / \mathrm{c}^{2}[19],[20],[21]$. This corresponds to $38 \mathrm{mrad}$ laboratory scattering angle for a $3.5 \mathrm{GeV} / \mathrm{c}$ antiproton scattered on a proton target (a liquid hydrogen scatterer will be used). The experiment can be done at the $24 \mathrm{GeV} / \mathrm{c}$ test beam at CERN impinging on a short (pointlike) solid target.

With a dipole magnet and a first straw tube tracker antiproton momenta and directions out of the production target are determined. Possible polarization effects are verified with a second straw tube tracker behind the liquid hydrogen analyzer. This results in an experimental setup as shown in figure 3 . The straw tube trackers provide an angular resolution better than $1 \mathrm{mrad}$ $[22]$.

In addition, an aerogel Cerenkov detector to discriminate the pion background and a scintillator hodoscope to generate a trigger signal have to be included. All these detectors stay in vacuum in order to reduce background and to avoid thick windows for the liquid hydrogen target.

A polarization determination with a precision below $2 \%$ is achievable within one week of beam time. A test beam of $4 \cdot 10^{11}$ protons every 2.4 seconds would produce about $4000 \bar{p} / \mathrm{s}$ within an acceptance range of e.g., the one of the CERN AD ring, whereby a target length reduction by a factor of 5 compared to the normal production target and a cut like indicated in figure 2 is taken into account.

The cross section of $1.35 \mathrm{mb}$ in the $t$-range $0.002-0.007$ [23] and a $15 \mathrm{~cm} l H_{2}$ analyzer target 
give about $3 \cdot 10^{5}$ useful analyzer scattering events/day resulting in a precision of 0.02 for the antiproton polarization within 3 days.

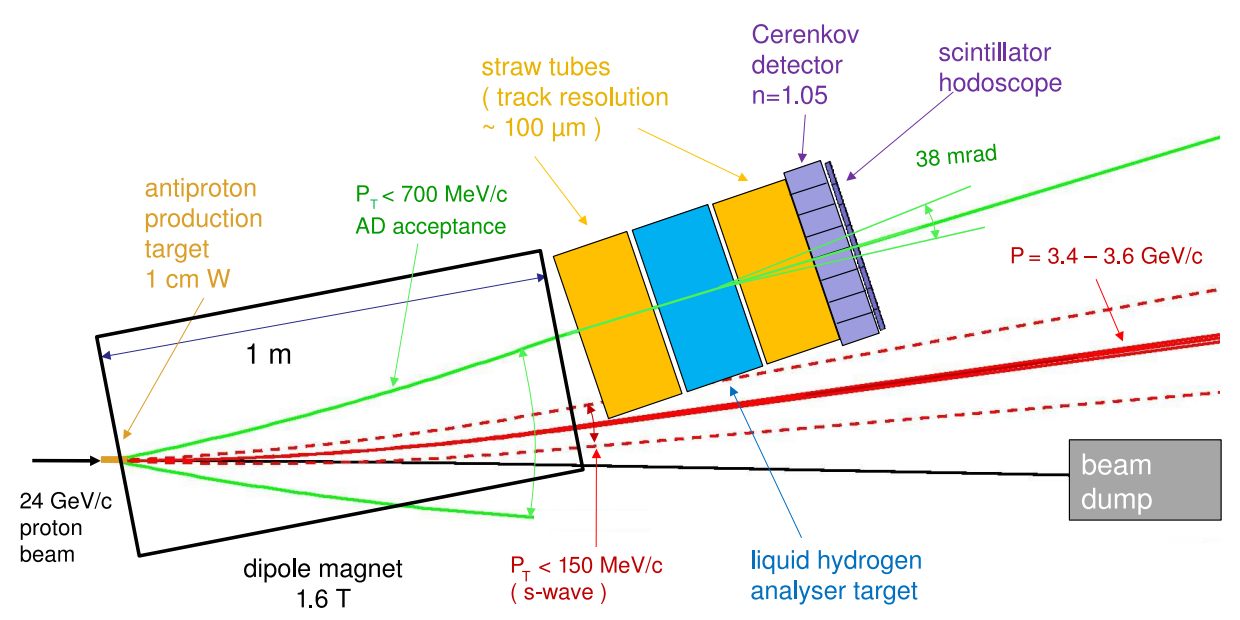

Figure 3. Experimental setup to measure the polarization of produced antiprotons.

Without the knowledge if and how much polarization is created in the production process no direct comparison to other methods can be made. The beam intensity at the $\bar{p}$ factory would be reduced by a factor of about 25 (target length reduction and cut in the angular range). Compared to the filter method no filtering time and no additional storage ring installations are needed. If nature is kind to us, the polarized antiproton production could be drastically simplified. But if there is no polarization observed, a further option would be to use a polarized proton beam for the antiproton production. There are at least some experimental hints that polarization is transferred to the produced antiproton as can be seen from analyzing power data of a $p p$ collider experiment with one beam polarized where produced antiprotons show a non-zero $A_{N}[24]$.

[1] Csonka P L et al 1968 Nucl. Instr. Meth. 63247

[2] Kilian K 1981 Symp. on HEP with polarized beams and targets, Lausanne 1980, Birkhäuser Verlag 219

[3] Kilian K and Möhl D 1982 Proceedings, Physics at LEAR, Erice, CERN/PS/LEA 701

[4] Rathmann F et al 1993 Phys. Rev. Lett. 711379

[5] Oellers D 2009 et al Phys. Lett. B 674269

[6] Schoch B 2010 Eur. Phys. J. A 435

[7] Lenisa P and Rathmann F for the PAX collaboration 2005 arXiv:hep-ex/0505054v1

[8] Barschel C et al (PAX collaboration) 2009 arXiv 0904.2325 [nucl-ex]

[9] Richard J M 1981 Symp. on HEP with polarized beams and targets, Lausanne 1980, Birkhäuser Verlag 535

[10] Dmitriev V F , Milstein A I and Strakhovenko V M 2008 Nucl. Inst. Meth. B 2661122

[11] Dmitriev V F, Milstein A I and Salnikov S G 2010 Phys. Lett. B690 427

[12] Proc. Workshop Bodega Bay, Krisch A D, Lin A M T and Chamberlain O (edts) 1986 AIP Conf. Proc. 145

[13] E. Steffens E 2008 AIP Conf.Proc. 9781

[14] E. Steffens E 2009 AIP Conf.Proc. 114980

[15] Meyer H O 2008 AIP Conf.Proc. 1008124

[16] Amsler C et al (Particle Data Group) 2010 J. Phys. G 3777 (the more precise value for $\Lambda$ decay is taken).

[17] Bravar A et al 1996 Phys. Rev. Lett. 772626

[18] Barnes P D et al 2000 Phys. Rev. C62 055203

[19] Buttimore N H et al 1999 Phys. Rev. D59 114010

[20] Grosnick D P et al 1990 Nucl. Instr. Meth. A290 269

[21] Okada H et al 2006 Phys. Lett. B 638450

[22] Wintz P for the COSY-TOF collaboration 2004 AIP Conf. Proc. 698789

[23] Armstrong T A et al 1996 Phys. Lett. B385 479

[24] Aidala C for the PHENIX and BRAHMS collaborations 2008 arXiv:0808.4139 [hep-ex] 